\title{
Ultrasound diagnosis of portal vein thrombosis following splenectomy
}

\author{
David Tudway ${ }^{1}$ and Graeme Sangster ${ }^{2}$ \\ ${ }^{I} X$-ray and ${ }^{2}$ Haematology Departments, East Birmingham Hospital, Bordesley Green East, Birmingham B9 5ST, \\ $U K$.
}

\begin{abstract}
Summary: We present a case of clinically unsuspected portal vein thrombosis occurring soon after splenectomy in a patient with chronic lymphocytic leukaemia. Ultrasound provided a means of easy and accurate diagnosis and allowed frequent reassessment of the thrombus during the period of its resolution under conservative management.
\end{abstract}

\section{Introduction}

Thrombosis of the portal venous system may occur for a variety of reasons although it has only rarely been reported as a complication of splenectomy (Ellison \& Fabri, 1983). It is thought to be more common after splenectomy performed for haematological disorders (Broe et al., 1981) although the reasons for this are not clearly understood. Portal vein thrombosis may be clinically silent but can be a serious condition with a high mortality.

The diagnosis may be difficult on clinical grounds alone but can be established by angiographic studies although these investigations are invasive and not without risk to the patient. The identification of portal venous thrombosis by ultrasound has been reported by Merritt (1979), Babcock (1979) and others. This technique has the advantages of being accurate, safe, readily available and easily repeated. Computed tomographic (CT) scanning is also of value (Vujic et al., 1980).

We report the value of ultrasound in demonstrating clinically unsuspected portal vein thrombosis in a patient with chronic lymphocytic leukaemia who had an otherwise unexplained pyrexia following splenectomy performed for hypersplenism. The early diagnosis allowed successful conservative management of this potentially serious problem and ultrasound was used to monitor resolution of the thrombosis.

\section{Case report}

A 53 year old Caucasian man presented in January 1983 with Stage C (Rai Classification) chronic lym-

Correspondence: D.C. Tudway, M.B., B.S., M.R.C.P., F.R.C.R.

Accepted: 20 June 1986 phocytic leukaemia. There was a partial response to treatment with cyclophosphamide, vincristine and prednisolone but hypersplenism persisted with splenomegaly, thrombocytopenia and bone marrow megakaryocytic hyperplasia although without erythroid hyperplasia. Splenectomy for hypersplenism was performed uneventfully in February 1985. The preoperative haemoglobin was $13.3 \mathrm{~g} / \mathrm{dl}$, white cell $44 \times 10^{9} / 1 \quad$ (lymphocytes $41 \times 10^{9} / 1$, neutrophils $1 \times 10^{9} / 1$ ), platelet count $93 \times 10^{9} / 1$. The spleen weighed $2670 \mathrm{~g}$ and was diffusely infiltrated by chronic lymphocytic leukaemia. Post-operatively the platelet count rose to $107 \times 10^{9} / 1$ on day 7 and reached a peak of $732 \times 10^{9} / 1$ on day 14 before falling to $411 \times 10^{9} / 1$ on day 30 and then stabilized around $250 \times 10^{9} / 1$ by the 90th day.

On the 2 nd day post-operatively a pyrexia of $38^{\circ} \mathrm{C}$ was noted although the patient was asymptomatic and clinical examination was normal. The chest $\mathrm{X}$-ray was normal; repeated cultures of sputum, urine, blood and wound swabs gave no bacterial growth. There were no clinical signs of peripheral deep venous thrombosis and abdominal examination was normal. The fluctuating low grade pyrexia persisted for 10 days despite flucloxacillin and ceftazidime. There was no biochemical evidence of liver dysfunction then or subsequently.

Abdominal ultrasound with a Siemens RAI $3.5 \mathrm{mHz}$ sector scanner performed 14 days after splenectomy showed no subphrenic fluid collection but extensive thrombus was seen in the stump of the splenic vein and in the portal vein. It could be seen that in several places the thrombus did not extend across the full width of the portal vein suggesting there might still be a channel allowing portal venous blood flow to continue (Figure 1). Thrombus extended up into the proximal intra- 


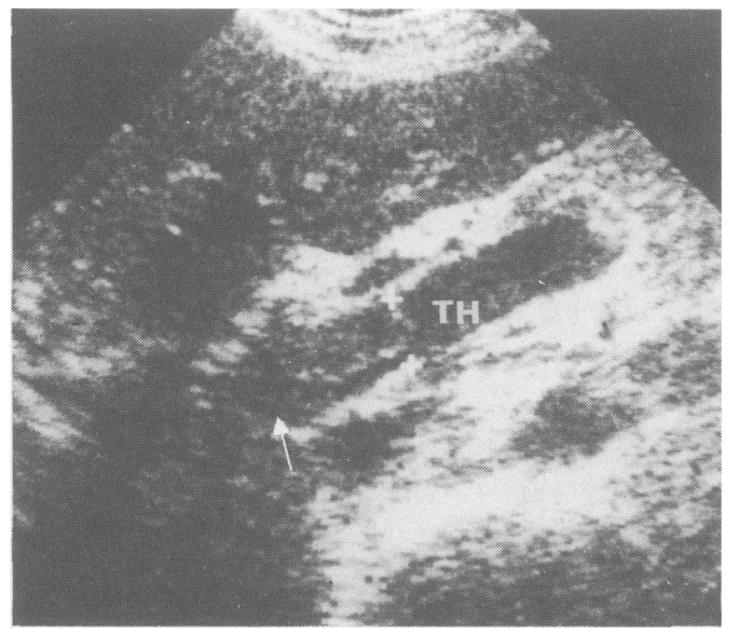

Figure 1 An ultrasound scan taken obliquely along the portal vein 14 days after splenectomy. The lumen is mostly filled with echogenic thrombus (TH) although there is a clear area (arrowed) close to the porta hepatis.

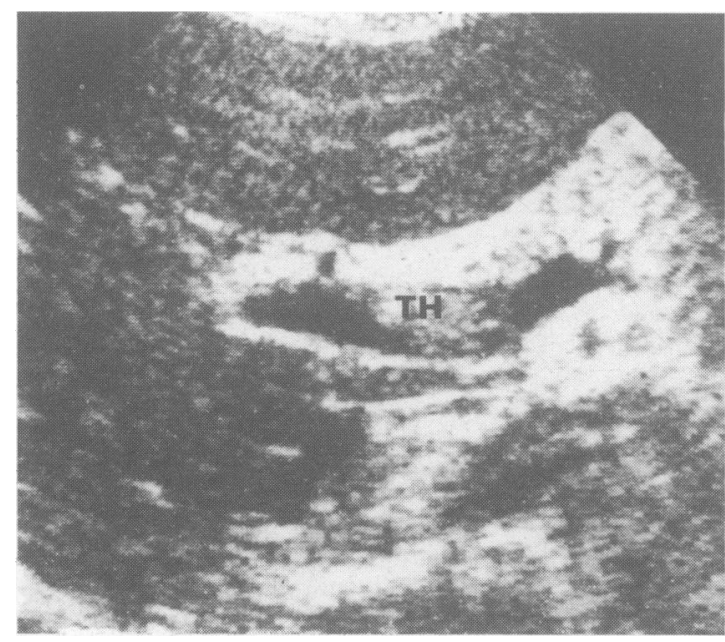

Figure 2 A similarly orientated scan to that in Figure 1 taken 7 weeks after splenectomy. The thrombus (TH) is greatly reduced but is still clearly visible in the portal vein.

hepatic portal branches but the superior mesenteric vein was entirely clear.

Following this the patient was anticoagulated with an intravenous heparin infusion for 14 days and then with warfarin for a total of 6 months. Subsequent ultrasound examinations, initially at weekly intervals, showed steady reduction in the size and extent of the thrombus (Figure 2) leaving only a small remnant in the main portal vein by 11 weeks after splenectomy. On all subsequent examinations the portal vein has been entirely clear of thrombus (Figure 3).

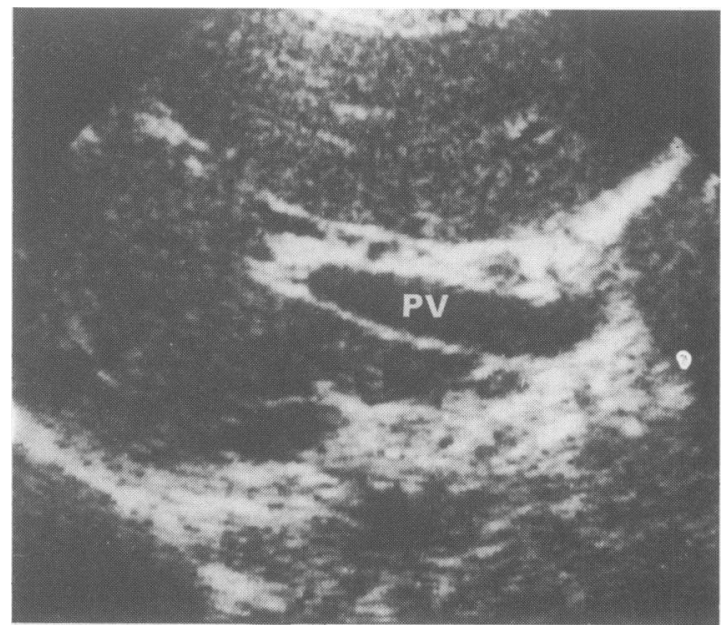

Figure 3 A similar scan of the portal vein to that in Figures 1 and 2 taken 6 months after splenectomy. The portal vein (PV) is clear of thrombus and is normal in appearance.

\section{Discussion}

Thrombosis in the portal venous system is most often a complication of intra-abdominal sepsis or malignancy but may occur rarely after splenectomy, usually i adult patients with a myeloproliferative disorder (Bre $\overrightarrow{0}$ et al., 1981; Bull et al., 1968; Balz \& Minton, 197 Gordon et al., 1978). It usually occurs within 6 week of splenectomy, as in our case, although later occurrences have been reported (Broe et al., 1981; Nagasue et al., 1977).

The clinical presentation of portal venous thrombosis is variable ranging from an almost complete absence of symptoms to an acute abdominal crisis. It may lead to the development of prehepatic portal hypertension and if there is associated mesenteric venous thrombosis the prognosis is poor and dependent on the degree and extent of bowel infarction (Carr \& Jamison, 1981).

Occlusion of the portal venous system causes a typical clinical presentation with abdominal pain, blood-stained diarrhoea and ascites. If the thrombus is non-occlusive, as in our case where portal vein thrombosis was unsuspected, it seems there may be little clincial evidence of its existence.

After splenectomy a thrombocytosis, defined as a platelet count $>400 \times 10^{9} / 1$, occurs, usually transient- $\tilde{\sigma}$ ly, in about $50 \%$ of patients. A causal relationship N between post-splenectomy thrombocytosis and $N$ thrombo-embolic complications has been proposed $\omega$ (Traetow et al., 1980) although even persistent extreme thrombocytosis (platelet count $>1000 \times 10^{9} / 1$ ) occur- $\stackrel{0}{C}$ ring in a variety of situations may be associated with $\stackrel{\odot}{\rightarrow}$ few thrombo-haemorrhagic complications (Buss et al., 
1985). Salter \& Sherlock (1957) and Boxer et al. (1978) showed that when splenectomy was incidental or performed for trauma, thromboembolic complications occurred no more frequently, even in the presence of thrombocytosis, than after any other abdominal operation. It is also noteworthy that thrombocytosis has been recorded in only about $25 \%$ of the reported cases of portal vein thrombosis following splenectomy. It appears, therefore, that thrombocytosis does not necessarily predispose to portal vein thrombosis. Abnormal platelet function and kinetics may increase the risk of thromboembolism, particularly amongst patients with myeloproliferative disorders undergoing splenectomy (Gordon et al., 1978). Another risk factor proposed by Hirsh \& Dacie (1966) is continuing anaemia with marrow erythroid hyperplasia after splenectomy which is thought to stimulate thrombopoiesis as well as erythropoiesis.

Venous stasis in the splenic vein remnant may also promote the development of thrombus in the portal venous system after splenectomy (Broe et al., 1981). The splenic vein may measure $3 \mathrm{~cm}$ or more when there is gross splenomegaly and unless divided close to its junction with the superior mesenteric vein a diverticulum with low blood flow remains. In the presence of damaged endothelium, particularly if there are also abnormalities of platelet function or numbers, thrombus may form and extend into mesenteric and portal veins.

In our patient portal vein thrombosis was successfully managed with anticoagulant therapy. Thrombolytic therapy or even surgical thrombectomy may be considered in the setting of progressive thrombosis and bowel resection is indicated when there is evidence of bowel infarction.

This case well demonstrates the ability of ultrasound to diagnose portal vein thrombosis. Subramanyan et al. (1984) could detect $94 \%$ of cases with ultrasound and $91 \%$ with angiography but only $76 \%$ with CT. The portal vein is visible in over $95 \%$ of abdominal ultrasound examinations (Merritt, 1979) and the splenic and superior mesenteric veins are

\section{References}

BABCOCK, D.S. (1979). Ultrasound diagnosis of portal vein thrombosis as a complication of appendicitis. American Journal of Roentgenology, 133, 317.

BALZ, J. \& MINTON, J.P. (1975). Mesenteric thrombosis following splenectomy. Annals of Surgery, 181, 126.

BOXER, M.A., BROWN, J. \& ELLMAN, L. (1978). Thromboembolic risk of post splenectomy thrombocytosis. Archives of Surgery, 113, 808.

BROE, P.J., CONLEY, C.L. \& CAMERON, J.L. (1981). Thrombosis of the portal vein following splenectomy for myeloid usually visible. Unlike angiography, ultrasound is harmless and non-invasive and can be repeated whenever required and, unlike CT, it allows total freedom of orientation of scans so that vessels can be imaged at will along their long axes and the full extent of any thrombus can be assessed. Ultrasound must be regarded as the diagnostic method of choice for portal vein thrombosis. The ultrasound characteristics of portal vein thrombosis are: thrombus seen within the portal vein, dilation of the splenic and superior mesenteric veins and loss of the normal portal venous landmarks (Merritt, 1979). Only the first of these features was definitely present in our case but this alone is diagnostic.

Our patient had an unexplained post-operative pyrexia and this was perhaps due to the portal vein thrombosis. Such a fever with no apparent infective or other cause has been described as a complication of splenectomy (Holme \& Crosby, 1984; Ellison \& Fabri, 1983). Mo-Wah \& McFadzean (1974) have suggested that fever can be due to circulating leucocyte agglutinating antibodies which are normally removed by the spleen. Holme \& Crosby (1984) encountered unexplained pyrexias after $10 \%$ of elective splenectomies performed mainly in Hodgkin's disease and lymphoma but also for a range of other haematological disorders. It is possible that some of these may have had undiagnosed non-occlusive portal vein thrombosis which may be a commoner complication of splenectomy than is realized. We consider that an ultrasound examination is indicated whenever there is a pyrexia of unknown origin following splenectomy or whenever portal vein thrombosis is suspected.

In conclusion, we have described a case of reversible portal vein thrombosis, an infrequently reported complication of splenectomy, and have documented its resolution with ultrasound. The diagnostic and clinical aspects of the case have been discussed.

\section{Acknowledgements}

We wish to thank Dr M.J. Leyland, whose patient this is, and Mrs J. Bull for typing the manuscript. metaplasia. Surgery, Gynecology and Obstetrics, 152, 488. BULL, S.M., ZIBRIA, B.A. \& FERRER, J.M. (1968). Mesenteric venous thrombosis following splenectomy: Report of two cases. Annals of Surgery, 162, 968.

BUSS, D.H., STUART, J.J. \& LIPSCOMB, G.E. (1985). The incidence of thrombotic and haemorrhagic disorders in association with extreme thrombocytosis: an analysis of 129 cases. American Journal of Hematology, 20, 365.

CARR, N. \& JAMISON, M.H. (1981). Superior mesenteric venous thrombosis. British Journal of Surgery, 68, 343. 
ELLISON, E.C. \& FABRI, P.J. (1983). Complications of splenectomy. Etiology, prevention and management. Surgical Clinics of North America, 63, 1313.

GORDON, D.H., SCHAFFNER, A.D., BENNETT, J.M. \& SCHWARTZ, S.I. (1978). Post splenectomy thrombocytosis: its association with mesenteric, portal and/or renal vein thrombosis in patients with myeloproliferative disorders. Archives of Surgery, 113, 713.

HIRSH, J. \& DACIE, J.V. (1966). Persistent post splenectomy thrombocytosis and thromboembolism: a consequence of continuing anaemia. British Journal of Haematology, 12, 44.

HOLME, T.C. \& CROSBY, D.L. (1984). Elective splenectomy. Indications and complications in 102 patients. Journal of the Royal College of Surgeons of Edinburgh, 29, 229.

MERRITT, C.R.B. (1979). Ultrasonographic demonstration of portal vein thrombosis. Radiology, 133, 425.

MO-WAH, C. \& McFADZEAN, A.J.S. (1974). Post splenectomy fever. Transactions of the Royal Society of Tropical
Medicine and Hygiene, 68, 437.

NAGASUE, N., INOKUCHI, K., KOBAYASHI, M. \& SAKU, M. (1977). Mesenteric venous thrombosis occurring late after splenectomy. British Journal of Surgery, 64, 781.

SALTER, P.P. \& SHERLOCK, E.C. (1957). Splenectomy, thrombocytosis and venous thrombosis. American Surgeon, 23, 549.

SUBRAMANYAN, B.R., BALTHAZAR, E.J., LEFLEUR. R.S., HORII, S.C. \& HULNICK, D.H. (1984). Portal venous thrombosis: correlative analysis of sonography, CT and angiography. American Journal of Gastroenterology, 79, 773.

TRAETOW, W.D., FABRI, P.J. \& CAREY, L.C. (1980). Changing indications for splenectomy. Archives of Surgery, 115, 447.

VUJIC, I., ROGERS, C.I. \& LEVEEN, H.H. (1980). Computed tomographic detection of portal vein thrombosis. Radiology, 135, 697. 